\title{
Ambiances
}

anbiances Environnement sensible, architecture et espace urbain Varia | 2013

\section{Contexte spatial des ambiances urbaines et usage des lieux}

Spatial context of urban ambiances and use of place

\section{Arnaud Piombini}

\section{(2) OpenEdition}

\section{Journals}

Édition électronique

URL : http://journals.openedition.org/ambiances/261

DOI : 10.4000/ambiances.261

ISSN : 2266-839X

\section{Éditeur :}

Direction Générale des Patrimoines - DAPA - MCC, UMR 1563 - Ambiances Architectures Urbanités $(\mathrm{AAU})$

\section{Référence électronique}

Arnaud Piombini, "Contexte spatial des ambiances urbaines et usage des lieux », Ambiances [En ligne], Varia, mis en ligne le 07 février 2013, consulté le 19 avril 2019. URL : http://

journals.openedition.org/ambiances/261; DOI : 10.4000/ambiances.261

Ce document a été généré automatiquement le 19 avril 2019.

\section{cc) (†)}

Ambiances is licensed under a Creative Commons Attribution-NonCommercial-NoDerivatives 4.0 International License. 


\title{
Contexte spatial des ambiances urbaines et usage des lieux
}

Spatial context of urban ambiances and use of place

\author{
Arnaud Piombini
}

\section{Introduction}

1 La géographie a notamment pour but de comprendre comment se construit le rapport entre individus et territoires. Or, au-delà de ce qui fonde l'existence même du territoire, l'appropriation d'une portion d'espace par un groupe humain, on trouve l'idéel, le sentiment d'appartenance, la relation à un espace en tant que lieu de vie et qui mobilisent des représentations cognitives, des relations sensibles. Ainsi, certaines notions comme la perception, la cognition, les représentations de l'espace ont émergé dans la discipline géographique. Ces thématiques permettent de mettre en relation les structures spatiales objectives et les significations sociales, culturelles et sensibles des lieux. La ville passe ainsi du concret au perçu, au représenté, de l'objectif au subjectif (Berque, 1995 ; Santos, 1997).

2 Les ambiances font justement référence à une expérience vécue sur le plan de la sensibilité. Elles se fondent sur une spatialité du proche, du contact, de l'interaction avec l'environnement immédiat, une géographie du vécu (Besse, 2010) qui fait écho à la territorialité des individus. Or, cette territorialité s'exprime à plusieurs échelles et si les ambiances sont multi-sensorielles, elles sont aussi multi-scalaires. Il paraît donc nécessaire de comprendre comment un espace, au travers de ses ambiances, se connecte à son environnement proche car si les caractéristiques des lieux font sens pour les individus, les appréciations portées sur les espaces ne peuvent être appréhendées hors contexte.

3 Dans cette optique, certaines méthodes de l'analyse spatiale peuvent être mobilisées pour mettre en évidence les organisations, les structures territoriales et ambiantales 
auxquelles les citadins sont confrontés et qu'ils vont nécessairement mettre «en mouvement» au cours de leurs déplacements. Cette notion de mobilité est centrale car elle va également nous permettre de préciser la manière dont les ambiances sont perçues. En effet, l'utilisation de l'espace urbain au quotidien et le choix des espaces fréquentés sont intimement liés à la perception des ambiances urbaines locales par les individus. D'ailleurs, on pourra presque parler de «rationalité affectuelle » pour expliquer l'action humaine au regard des qualités sensibles des espaces et des émotions et affects qu'ils génèrent (Audas \& Martouzet, 2008).

Dans cet article, plusieurs questions essentielles seront traitées. Comment l'organisation spatiale, abordée ici par le prisme des ambiances urbaines, s'impose-t-elle aux individus et introduit de l'hétérogénéité spatiale ? Comment les individus vont-ils s'accommoder de cette contrainte en fonction de leur contexte de localisation? De quelle manière ces choix vont-ils exprimer des préférences ambiantales? Pour répondre à ces interrogations, la première partie décrira quelques méthodes classiques de géostatistique qui s'appliquent parfaitement à la description de la configuration spatiale des lieux et de leurs ambiances. Dans un second temps, nous nous intéresserons au contexte spatial des individus dans le cadre de leurs déplacements pédestres. Cette étape sera l'occasion de mobiliser un modèle de choix peu utilisé dans les travaux sur les ambiances et paysages urbains et qui permet de prendre en compte les contextes spatiaux pour identifier les préférences ambiantales des citadins.

\section{Appréhender le contexte spatial des ambiances urbaines}

\section{Ambiances et paysages, éléments particuliers de la relation des individus à l'espace}

5 La notion d'ambiance repose sur l'ensemble des sens humains et se décrit comme la rencontre entre une donnée physique et ce que les sens en perçoivent (Augoyard, 1979). Cette thématique des ambiances peut renvoyer à l'étude des singularités, des spécificités par nature difficiles à appréhender et à généraliser. Elle fait notamment référence à la notion de paysage qui est un objet d'étude important en géographie; historiquement, la lecture du paysage permettait d'identifier les spécificités des espaces pour délimiter des régions homogènes. Mais les travaux de $\mathrm{K}$. Lynch ont permis de renouveler cette approche et d'insister sur la dimension visuelle de la perception humaine qui doit favoriser la création d'images mentales, individuelles et collectives de l'espace (Lynch, 1969). Ainsi, le paysage existe «dans sa relation avec un sujet dont la conscience et l'émotion singulières transforment l'objectivité sensorielle du regard (le monde que nous voyons presque tous de la même façon) et conduisent aux représentations qui en découlent » (Brossard \& Joly, 2004, p. 23).

6 Le paysage n'existe donc qu'à travers chaque regard; on retrouve ici des principes phénoménologiques au sens de Husserl puisque l'on s'attache à décrire l'expérience telle qu'elle émerge dans une situation donnée (Besse, 2010 ; Thibaud, 2002), telle qu'elle est vécue par chaque individu en fonction de sa subjectivité. On parlera de paysage perçu, de connotation subjective des lieux (Sansot, 1973), d'évaluation affective des paysages (Wong 
\& Domroes, 2005) qui émergent au contact direct des lieux mais cela ne doit pas occulter le contexte spatial plus large dans lequel ambiances et paysages s'inscrivent.

\section{Échelles et mesures des configurations spatiales d'ambiances}

7 D'un point de vue spatial, la question des échelles est centrale, notamment en termes d'aménagement, d'environnement construit. D'ailleurs, la vocation de la ville a toujours reposé sur la confrontation, dans un même espace, de phénomènes associés à des échelles très variées (Gourdon, 2001). Même si les ambiances sont compréhensibles dans l'instant, dans l'immédiateté de la relation homme-espace, elles doivent être lues à plusieurs niveaux. La notion de milieu, très utilisée en géographie, désigne ce qui est autour du lieu, mais dans quoi il est plongé (Brunet et al., 1992). Par extension, on retiendra que le milieu peut se définir comme le contexte signifiant, autant du point de vue du cadre de vie que de l'action humaine (Sack, 1997 cité par Labussière, 2009). Ainsi, pour les citadins en mouvement, chaque attribut dans l'espace agit tout autant par sa distribution spatiale, le rythme de ses intensités que par ses propriétés élémentaires (Merleau-Ponty, 1942). Ce faisant, on remet le comportement des individus en perspective, au-delà de ce que l'on peut relever dans des lieux singuliers; on va chercher dans les formes d'organisation des espaces les raisons d'une préférence exprimée localement. Sur la base de cette assertion, il est pertinent d'étudier le lien environnement sensible - usage des lieux à différents niveaux scalaires.

8 A un niveau micrologique, on s'intéresse à l'environnement immédiat qui entoure l'individu dans l'espace public. On cherche alors à définir des ambiances en un lieu ce qui impose une discrétisation de l'espace avec des limites plus ou moins artificielles, fréquemment déterminées selon des règles morphologiques. Dans la théorie de la syntaxe spatiale par exemple, les espaces convexes ou lignes axiales sont définis à cette échelle sur la base de règles d'inter-visibilité (Hillier et Hanson, 1984). Ces portions d'espaces se caractérisent par un continuum visuel et peuvent donc être appréhendées instantanément par les individus. Ce type de découpage en unités spatiales va souvent servir de base à la mise en œuvre de mesures d'ambiances. Le niveau micrologique est donc essentiel dans la mesure où il rend intelligibles les espaces urbains en les discrétisant et en les qualifiant. A ce niveau, les ambiances conjuguent objectif et subjectif; elles permettent de questionner les relations entre conception des espaces (formes construites, objets matériels mesurés...) et expérience sensorielle des individus (perceptions, représentations, usages...).

9 Cette étape nécessaire doit toutefois être complétée par des mesures d'intégration de ces unités spatiales de base au reste de la ville. En examinant les relations entre ces unités, on replace la question des ambiances au niveau des citadins et de leurs usages qui, par leurs déplacements, vont lier ces échelles micrologiques entre elles. A ce niveau mésologique, on s'intéresse à la configuration spatiale qui s'impose en partie aux individus; les formes urbaines deviennent prégnantes par rapport aux formes construites plus locales. On va alors interroger la structure spatiale pour comprendre comment elle peut être mise en mouvement par les citadins en fonction des localisations relatives des ambiances urbaines (dynamique sensori-motrice). Pour cela, il faut commencer par définir des relations de voisinage entre les unités spatiales. Ces relations peuvent être relevées par contact direct (contiguïté d'ordre 1 - illustration 1) mais l'on peut aussi s'affranchir de cette continuité spatiale en établissant des liens entre des unités elles-mêmes séparées par d'autres unités 
(une contiguïté d'ordre 2 signifie qu'il faut traverser une unité spatiale pour relier deux ensembles).

Illustration 1 : unités spatiales et contiguïtés d'ordre 1

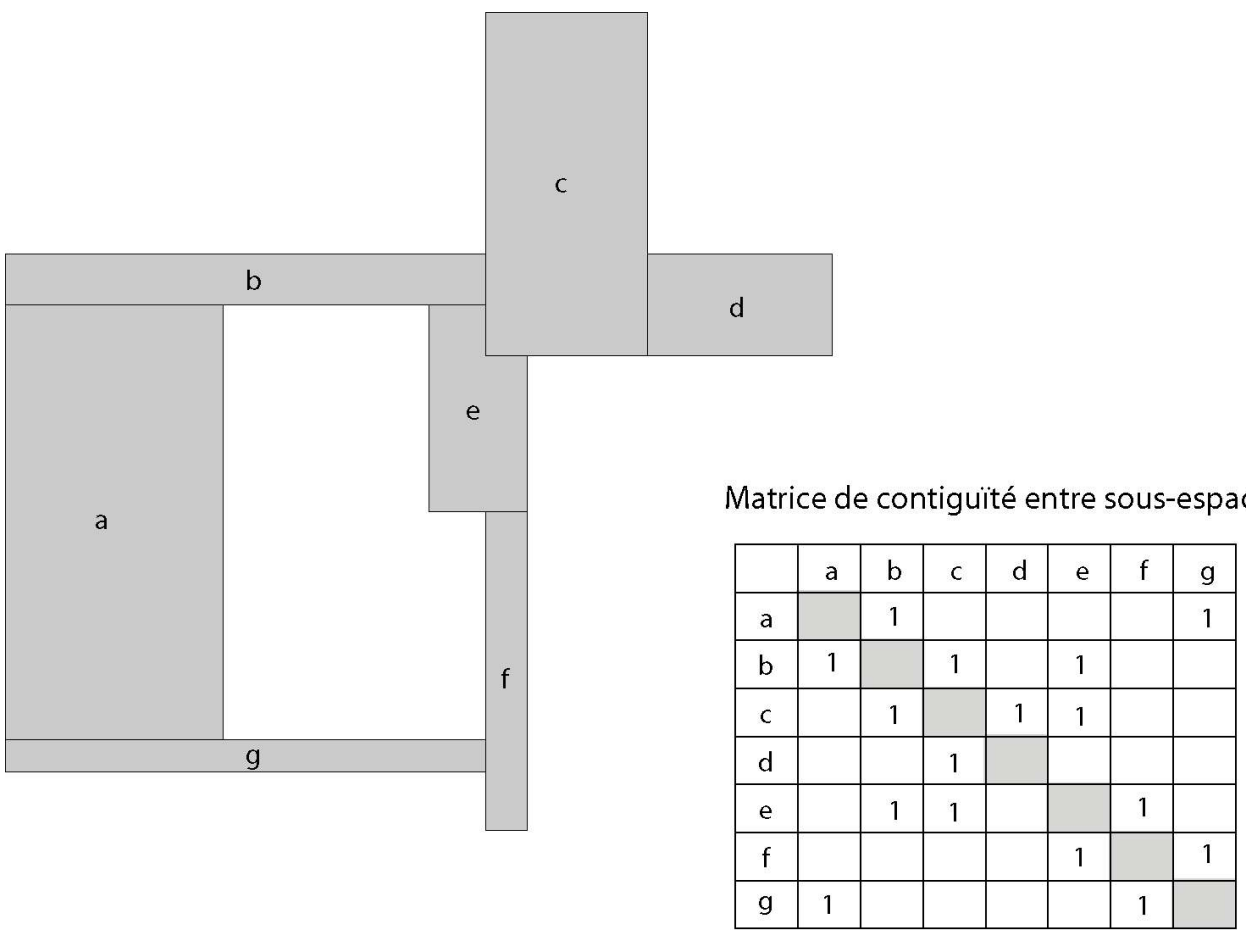

10 Avec ces différents niveaux de contiguité, la question des ambiances va être traitée à des échelles variées : on pourra parler d'ambiances «instantanées » dans un espace donné, d'ambiances de proximité qui sont connectées au lieu dans lequel se trouve une personne et d'ambiances potentiellement accessibles qui sont à une certaine portée et sont éventuellement prises en considération par l'individu. Plusieurs méthodes d'analyse géostatistique permettent de mettre en évidence les relations entre les unités spatiales à ces différentes échelles.

\section{L'entropie spatiale}

11 L'entropie de Shannon appliquée en géographie mesure le degré de désordre de la distribution spatiale d'un indicateur donné. Concernant la thématique des ambiances, on pourra mesurer la distribution d'un indicateur dans l'espace pour mettre en évidence des phénomènes de concentration ou de dispersion sachant que l'on peut faire varier le niveau d'analyse de l'intérieur d'une unité spatiale à l'ensemble de la ville.

Si la valeur de l'entropie tend vers 0 , cela signifie que des concentrations spatiales émergent et qu'il se dégage une structure spatiale spécifique. On pourra alors parler d'hétérogénéité spatiale, d'une certaine inégalité ambiantale selon le lieu où l'on se trouve. En revanche, si la valeur de l'entropie tend vers 1, cela indique que la variable ambiantale est distribuée de manière uniforme; les espaces se caractérisent par une composition relativement similaire. On parlera alors d'homogénéité spatiale.

Cette entropie ne permet cependant pas de donner une indication sur le degré de contrainte de l'espace. En effet, on peut considérer que, d'un extrême à l'autre, 
l'homogénéité parfaite d'un espace tout comme une hétérogénéité très forte ne donne pas aux individus de réelles possibilités de modulation et donc de choix des ambiances auxquelles ils seront confrontés. En outre, si l'indice d'entropie permet de mesurer l'organisation d'une structure spatiale donnée, il ne nous informe pas sur les liens locaux entre sous-espaces.

\section{L'autocorrélation spatiale}

14 L'autocorrélation spatiale permet de mettre en évidence des portions d'espaces voisines pour lesquelles une certaine homogénéité émerge (clusters spatiaux). Elle mesure la corrélation d'une variable d'ambiance donnée avec elle-même, selon des règles de proximité spatiale. Ainsi, l'indice de Moran propose une mesure globale pour un ordre de contiguité donné. L'intérêt réside notamment dans la possibilité de tester plusieurs niveaux de voisinage afin de mesurer le degré de similarité ambiantale d'un sous-espace à ses voisins pour le comparer à l'ensemble des sous-espaces de la ville. Malgré son intérêt pour la description globale d'un espace, l'indice de Moran ne permet pas directement de mettre en évidence les structures spatiales locales qui peuvent émerger dans la ville et qui sont susceptibles d'affecter les citadins.

Pour remédier à cette carence, l'indice locale d'association spatiale (LISA) a été proposé par L. Anselin et constitue la version locale et décomposée de l'indice de Moran (Anselin, 1995). Cet indice présente également l'avantage de pouvoir être cartographié. L'illustration 2 propose des relevés de terrain de présence de végétation par unités spatiales, ici des tronçons de rues (élément du réseau viaire entre deux carrefours). La cartographie des résultats montre une structure spatiale spécifique mais une tout autre configuration peut également être mise en évidence par des calculs d'autocorrélation.

L'autocorrélation spatiale locale s'interprète de la manière suivante. Si la valeur d'un indice ambiantal dans un espace est relativement similaire aux valeurs relevées dans les zones voisines, il existe un effet de la structure spatiale, les ambiances se caractérisent par une autocorrélation spatiale positive. Cela peut s'interpréter, pour les citadins en mouvement, comme une continuité ambiantale locale. Cette continuité qui s'exprime par des propriétés identiques d'un espace à l'autre offre finalement peu de variations de choix pour les individus ce qui suppose peut-être une certaine " monotonie ambiantale ». En revanche, en cas d'autocorrélation négative, des unités spatiales voisines présenteront des ambiances différentes, les valeurs élevées et faibles alternent pour un indice donné. L'interprétation qui peut en être faite montre certes une diversité mais aussi une discontinuité ambiantale, des ruptures d'ambiances qui s'imposent à l'usager en mouvement. Dans le cas d'une absence d'autocorrélation, aucune structure spatiale précise n'émerge.

Sur l'illustration 2, on remarquera que l'information d'ambiance ne repose plus sur la végétation elle-même mais plutôt sur la manière dont les niveaux de végétation s'articulent entre eux. Ainsi, on peut voir émerger des autocorrélations fortes en des lieux où il n'y a pas du tout de végétation ce qui indique une homogénéité locale. Par ailleurs, les perspectives visuelles pour lesquelles il y a une forte soumission à la végétation (on peut presque parler ici de corridor vert) ne sont pas forcément caractérisées par une autocorrélation élevée. Cela s'explique par le fait que les tronçons composant la perspective sont connectés à des espaces transversaux différenciés. 

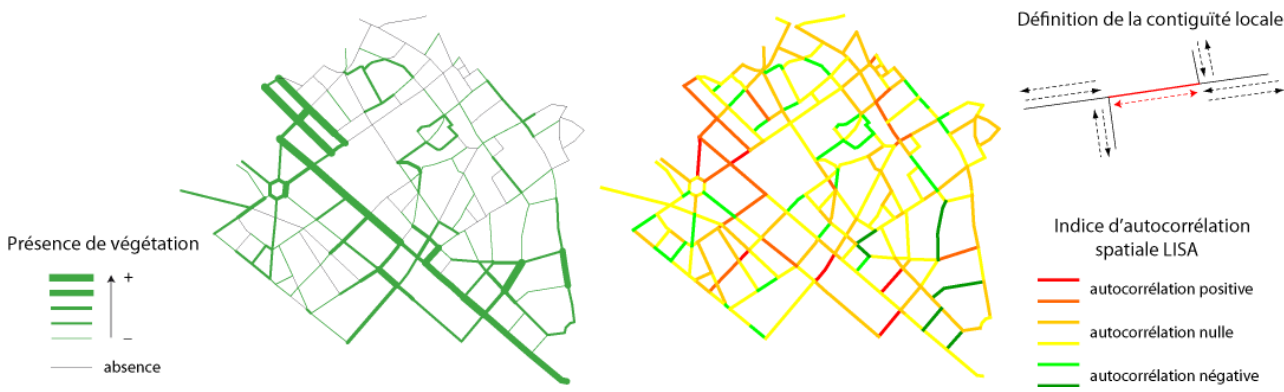

Centre-ville de Lille qui permettent d'affecter à un espace une valeur ambiantale agrégée calculée sur la base des valeurs relevées dans l'espace en question et dans les unités spatiales qui lui sont connectées. Cet indicateur est également très intéressant car il s'appuie sur l'hypothèse de contexte selon laquelle la valeur accordée à un espace par un individu dépend des ambiances perçues in situ ainsi que sur celles des espaces environnants par effet de contiguïté ou de proximité (Gourdon, 2001). Des mesures de fragmentation ou de connectivité d'ambiances, utilisées par exemple en écologie du paysage, peuvent également compléter les indices présentés ci-dessus. L'indice de connectivité pourrait par exemple mettre en évidence la perspective visuelle de soumission à la végétation ignorée par la mesure d'autocorrélation spatiale dans l'illustration 2.

Bien entendu, ces mesures sont très dépendantes de la délimitation des sous-espaces étudiés, de l'échelle d'analyse retenue ainsi que de l'ordre de contiguité choisi. Mais elles peuvent fournir des indications très intéressantes sur la disposition spatiale des ambiances et donc sur la contrainte structurelle que la ville impose aux individus.

\section{Les usages des espaces urbains}

Comme nous l'avons vu, le contexte des ambiances urbaines peut être révélé par un certain nombre d'indicateurs spatiaux. Mais pour comprendre ce qu'en font les individus en termes de choix, d'évitement, de franchissement des lieux, il est nécessaire d'étudier les stratégies de déplacement.

\section{Le choix de la mobilité pédestre}

21 Le concept d'adhérence, qui symbolise pour chaque individu et en fonction du mode de déplacement choisi le degré d'interaction avec l'espace du mouvement (Amar, 1993), distingue la mobilité pédestre des autres modes parce qu'elle induit, pour celui qui la pratique, une immersion complète dans l'environnement. La marche à pied induit un contact par les sens avec l'environnement immédiat de déplacement (Amar, 1993). Ainsi, le piéton est un véritable capteur multi-sensoriel et, à ce titre, les liens qui l'unissent aux espaces urbains sont d'un grand intérêt. Ce mode de déplacement semble donc parfaitement adapté à l'étude des ambiances urbaines car le piéton est, en outre, un véritable spécialiste d'une partie de sa ville et les choix d'usages qu'il va mettre en œuvre sont potentiellement d'une grande richesse interprétative. $R$. Thomas propose ainsi « une 
ethnographie sensible de ces expériences déambulatoires " qui permet de décrire et d'analyser les mobilités (Thomas, 2004, p.3). L'objectif pourrait consister à mettre en évidence les facteurs sensibles influençant les micro-mobilités piétonnes (Besse, 2010) en prenant en compte les effets potentiels des structures spatiales.

Certes, on pourra objecter que le « capteur humain piéton » est imparfait du point de vue de la stricte objectivité : sa connaissance de la ville est partielle, ses filtres perceptifs, ses représentations sont éminemment personnelles et dépendantes des motivations, des intentions, des projets associés à l'action (Di Méo, 1991 ; Golledge \& Stimson, 1997). In fine, le déplacement d'une personne repose sur une combinaison complexe de connaissances et de préférences individuelles (Paulet, 2002). Mais par la multiplication des observations individuelles, on peut faire émerger une compréhension collective des pratiques urbaines en détectant les espaces fonctionnels sélectionnés au sein de l'espace réel (Cauvin, 1999). Ce point est essentiel car il peut être rapproché de méthodes d'analyse des choix individuels issues de l'économie et utilisées en géographie (Foltête \& Piombini, 2010).

\section{Analyse des comportements de mobilité}

Le piéton a une appréhension sensitive de la ville et en même temps relativement rationnelle. La rationalité pointe ici le fait que le piéton ne marche pas au hasard dans la ville dans le cadre de ses déplacements quotidiens (minimisation du temps, de la distance parcourue...). Cette rationalité s'exprime de manière large : à une minimisation du temps ou des distances parcourues, le piéton va, en distinguant les qualités et défauts des lieux potentiellement accessibles, chercher à maximiser la fréquentation des espaces attractifs aux dépens de ceux qui le sont moins. Certes, l'individu « a moins appris à connaître le monde qu'il ne s'est exercé à y faire face » (Goffman, 1973, p. 237) mais il sait aussi éviter les occasions, les lieux, les activités et les objets qui nécessiteraient des efforts d'adaptation trop conséquents (Goffman, 1973). C'est ce choix, non dénué de sens, qu'il convient d'étudier car il peut révéler les préférences d'ambiances des individus.

Cette approche dite des préférences révélées s'oppose aux enquêtes plus traditionnelles basées sur les déclarations des individus. Une méthode classique consiste par exemple à travailler sur des parcours commentés pour caractériser les modes d'appréhension des phénomènes d'ambiance ainsi que les significations que leur donnent les individus (Péneau, 2000). Aussi intéressants que soient ces travaux, il faut noter que les personnes ne sont pas toujours capables d'analyser objectivement et de manière fiable les raisons de leurs choix (Moessinger, 1996). R. Golledge l'a démontré au sujet des choix d'itinéraires en comparant les affirmations d'enquêtés et ce que leurs trajets révélaient (Golledge, 1999). Si les individus sont souvent capables d'exprimer le sens et l'intensité des relations qui les lient aux environnements urbains (aversion, rejet, tolérance, indifférence) (Di Méo, 1991; Depraz, 2005), ces derniers n'ont pas toujours conscience des critères qui motivent leurs choix (Costermans, 2001) et l'on ne peut espérer recueillir des discours complètement objectifs de la part des personnes interrogées (Loiseau et al., 1993).

Une analyse des espaces fréquentés doit permettre d'alimenter ce questionnement sur l'identification des préférences environnementales. A partir de plusieurs ambiances associées à des lieux « figés » qui sont le résultat de l'aménagement urbain, le piéton va composer parmi un ensemble des possibles. Il va choisir son espace vécu au sein d'un espace ressource non neutre qui, comme nous l'avons vu, offre des potentialités et exerce des contraintes. Chaque individu dispose d'un capital, d'un budget spatial présentant une 
grande variété de solutions de déplacements, variables selon le moment, le lieu ou le type de déplacement (Moles \& Rohmer, 1982). Ce capital n'est jamais consommé en totalité et des choix, non aléatoires et par conséquent interprétables, sont effectués. Ainsi, l'objectif est de comprendre comment l'on passe d'un espace structurel à un territoire " événementiel », choisi, pratiqué (Caron \& Roche, 2001).

\section{Influence du contexte spatial sur les choix}

Dans cette optique d'analyse des choix effectués, il faut relever l'importance du contexte spatial qui est très différencié d'un individu à l'autre. Tout l'espace n'est pas également accessible et, surtout, en fonction de la localisation dans l'espace, la variété des ambiances disponibles n'est qu'une réduction de l'ensemble des ambiances urbaines potentielles (illustration 3 ).

Illustration 3 : espace accessible et ambiances disponibles pour deux trajets distincts
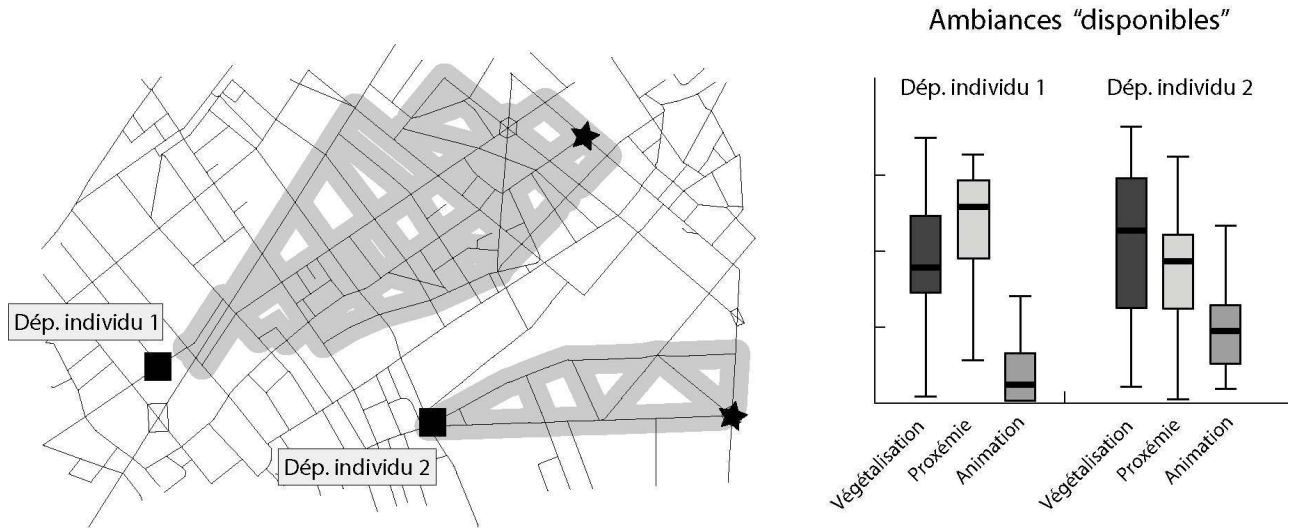

De ce point de vue, l'espace est très inégal puisque certains lieux se caractérisent par une grande diversité d'ambiances et donc d'options de choix alors que d'autres sont beaucoup plus homogènes. Les travaux sur la spatialisation des ambiances dans la ville, sur leurs localisations relatives (autocorrélation par exemple) permettent en théorie de prendre en compte ces spécificités dans le cadre des usages. Mais dans la réalité, la multitude des situations individuelles impose d'aller au-delà des mesures de configurations spatiales pour utiliser des méthodes d'analyse plus spécifiquement adaptées aux stratégies de déplacement des citadins. Les connectivités ambiantales potentielles mesurées par la structure déterminent une plus ou moins grande liberté théorique de choix des individus mais la connectivité effective ne peut être abordée que par l'analyse du réseau viaire accessible dans le cadre de chaque déplacement. Il n'est en effet pas possible d'anticiper le ressenti d'ambiances des individus sur la base d'un espace figé puisque celui-ci dépend des pratiques de déplacements qui vont permettre de composer une ambiance propre à chaque individu en fonction des caractéristiques disponibles. Ainsi, rares sont les usages des lieux strictement identiques puisqu'il existe une quasi infinité de combinaisons possibles. En revanche, il est possible de s'appuyer sur les choix effectués pour comprendre la perception des individus.

A cet effet, les modèles de choix discrets permettent de travailler de manière désagrégée sur les choix effectués par les personnes. Plutôt que de considérer une population dans sa globalité en mesurant des niveaux d'attraction des espaces urbains, les préférences 
exprimées par les individus sont analysées dans le contexte spécifique de leur déplacement.

\section{Un modèle ambiantal de choix discret}

Le modèle logit multinomial (McFadden, 1974) fait partie de la famille des modèles économiques de choix discrets et permet, par exemple, d'étudier les choix d'itinéraires des piétons parmi un ensemble d'alternatives potentielles discriminées sur la base de caractéristiques ambiantales. Comme nous l'avons mentionné, les contextes de choix sont très variables d'un individu à l'autre, en fonction de la localisation du déplacement sur le réseau. Or, les modèles de choix discrets fonctionnent sur la base de ces options disponibles et des caractéristiques qui leur sont associées. Dans ce cadre, ils permettent de mesurer précisément le rôle des ambiances urbaines. Le fonctionnement du modèle logit multinomial impose de disposer de données inhérentes aux choix de fréquentation des espaces effectués par les citadins, par exemple des trajets à pied dont les itinéraires ont été précisément relevés. Il est aussi nécessaire de caractériser les itinéraires à l'aide d'indicateurs morphologiques, d'ambiances, de paysages... La mise en œuvre du logit multinomial conduit ensuite à reproduire le processus de choix des individus qui peut être décrit comme suit :

- Génération des alternatives qui constituent l'ensemble de choix réduit considéré par l'individu ;

- Évaluation des attributs pertinents permettant de discriminer les alternatives;

- Choix de l'individu.

La première étape consiste à recenser, à partir d'un algorithme de calculs d'itinéraires, toutes les alternatives potentielles pour chaque trajet enregistré lors d'une enquête. Ce contexte de choix théoriquement pris en compte par les piétons correspond à tous les itinéraires raisonnables pour joindre l'origine à la destination. Ainsi, au-delà d'un certain allongement, les itinéraires (et donc les tronçons qui les composent) ne sont pas inclus dans l'ensemble de choix à partir duquel fonctionne le modèle. Dans un deuxième temps, l'objectif du modèle consiste à reproduire et donc expliquer le choix d'itinéraire effectué par chaque piéton par le calcul désagrégé de probabilités. Dans ces modèles, l'influence d'un indicateur d'ambiance donné est fonction de la distribution de ses valeurs au sein des chemins disponibles pour chaque trajet considéré. Ce modèle est dérivé de la théorie du consommateur en économie selon laquelle chaque individu est supposé agir de manière à maximiser sa satisfaction, en fonction des options de choix dont il dispose. Le logit multinomial prend donc en compte non seulement l'alternative choisie, mais aussi toutes celles refusées par chaque individu (illustration 4), ce qui permet d'analyser le choix en fonction du contexte et donne ainsi accès à une exploitation efficace des données d'ambiances. 


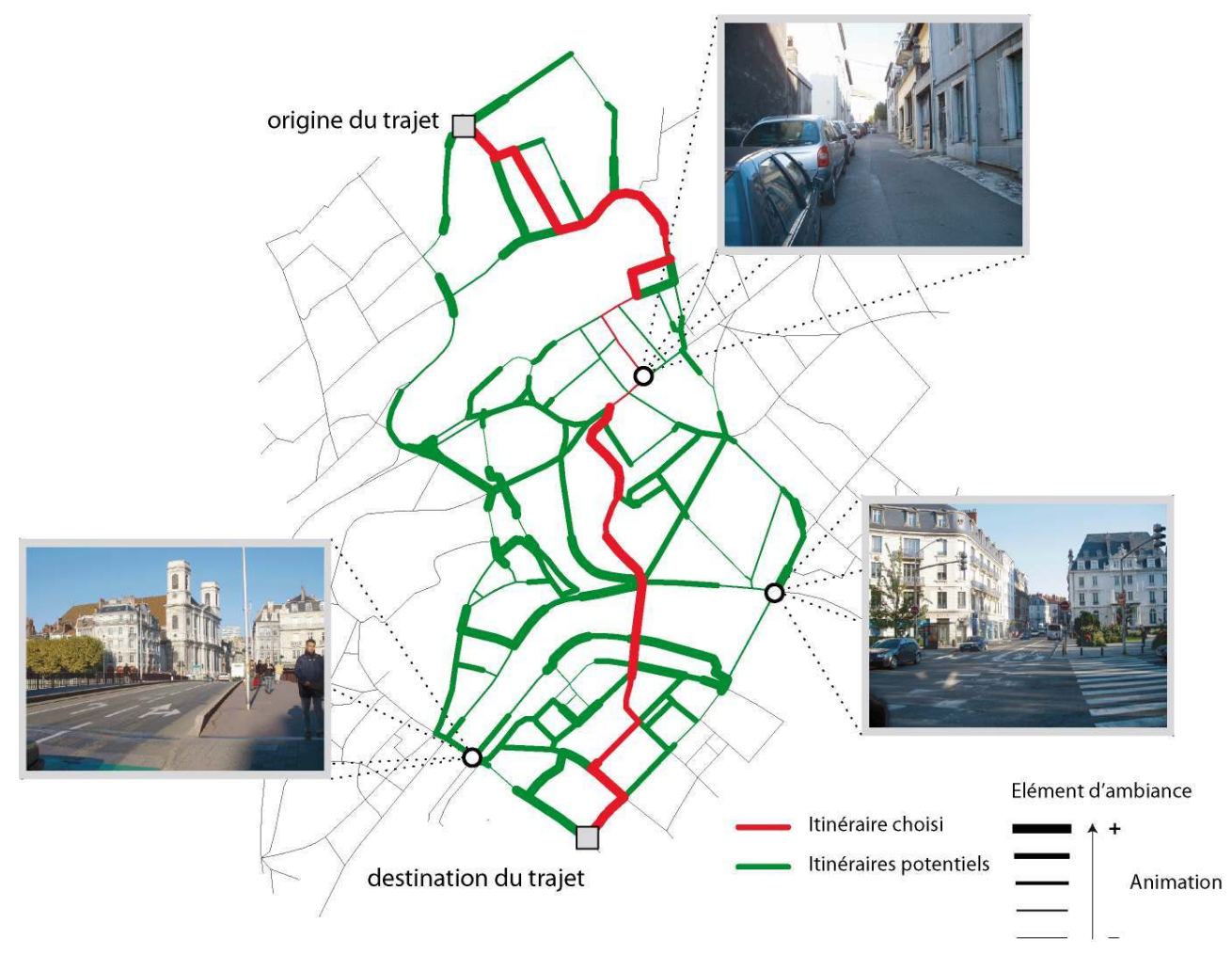

Besançon, quartier des Chaprais et centre-ville

31 La finalité du modèle est de reproduire les choix effectués par les piétons sur la base des caractéristiques d'ambiances mesurées pour chaque itinéraire. Les résultats obtenus se présentent sous la forme d'un vecteur d'attributs d'ambiances significatifs auxquels sont attachés des coefficients qui indiquent le sens de la relation et son intensité.

Prenons un exemple concret dans le quartier de Hautepierre à Strasbourg où de nombreux indicateurs morphologiques ont été calculés à partir des empreintes au sol des bâtiments et des espaces verts disponibles (BD Topo ${ }^{\oplus}$ de l'IGN). Ces indicateurs ont été construits sur la base des isovists qui sont des polygones formés par l'ensemble des points de l'espace visibles depuis un lieu d'observation donné. Finalement, trois d'entre eux se sont révélés pertinents pour expliquer les choix d'itinéraires des habitants (Woloszyn et al., 2011) :

- L'aire des isovists, pondérée négativement ;

- Le ratio de surfaces végétales inscrites dans chaque isovist qui exerce une influence très positive ;

- L'étirement de chaque isovist. Cette grandeur est pondérée positivement.

Dans ce contexte bien spécifique, les résultats obtenus montrent que les espaces intimes bornés par des bâtiments qui bloquent la visibilité sont davantage recherchés que les grands espaces ouverts. En revanche, l'attrait pour de longues perspectives visuelles confirme des hypothèses déjà largement éprouvées par les spécialistes de la syntaxe spatiale et du mouvement naturel (par exemple Hillier, 1996). L'influence positive du ratio de végétation démontre que les espaces verts constituent un élément important d'aménagement urbain et de valorisation des lieux aux yeux des citadins. 
Sur la base de ces résultats, il est possible de calculer, pour chaque tronçon, un potentiel d'attraction et de répulsion des itinéraires pédestres. Ce potentiel est fonction des coefficients d'ambiances retenus et des valeurs mesurées pour ces indices à l'intérieur de chaque tronçon. L'illustration 5 présente les résultats obtenus: en rouge figurent les tronçons attractifs et en bleu les tronçons répulsifs, l'épaisseur du tracé donnant une indication sur l'intensité des attractions / répulsions. Certaines zones concentrent des tronçons fortement attracteurs, ce qui signifie que nous avons affaire à des lieux dont les caractéristiques morphologiques correspondent aux indices mis en évidence par le modèle. Inversement, on voit émerger des espaces plus problématiques et qui seront potentiellement moins fréquentés.

Illustration 5 : carte de potentiel d'attraction des espaces urbains

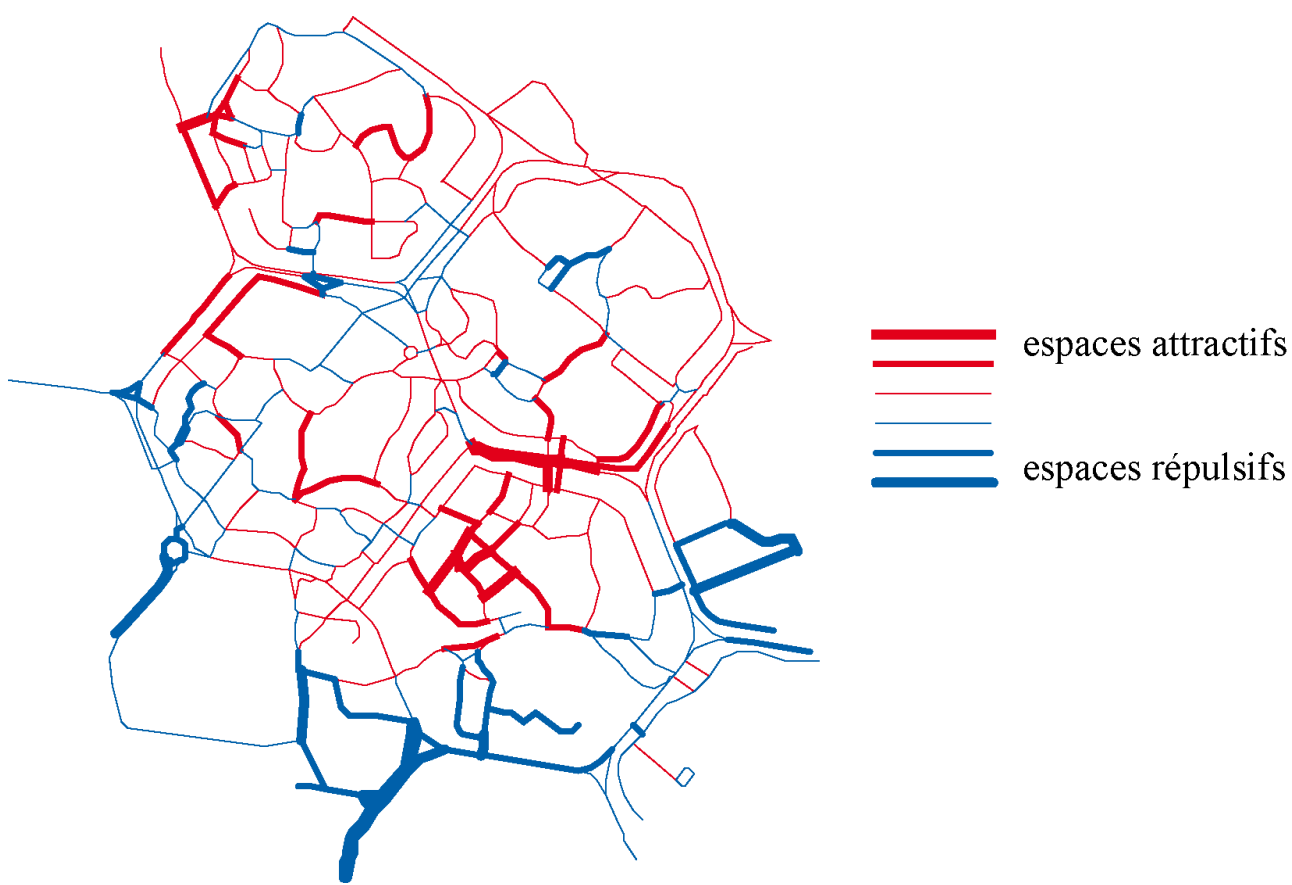

Strasbourg, quartier de Hautepierre

Ces résultats présentent des potentiels d'attraction mais cela ne signifie pas que ces tronçons soient nécessairement usités dans le cadre des parcours pédestres recensés lors de l'enquête. L'inverse est également vrai puisqu'un tronçon déprécié peut tout à fait être emprunté. Nous sommes bien, dans ce cas, en présence d'un potentiel structurel d'attraction. Suivant cette logique, on pourra comprendre qu'un lieu caractérisé par une ambiance potentiellement attractive ne sera pas nécessairement fréquenté par les citadins s'il est entouré d'espaces dépréciés. Il pourrait d'ailleurs être intéressant de proposer une mesure de fragmentation des espaces attractifs pour évaluer la possibilité « réelle » pour les citadins de relier ces espaces. De manière opposée, certains travaux sur la cognition spatiale qui portent sur les stratégies de déplacement montrent qu’à « une stratégie locale, où les utilités des divers mouvements possibles reposent exclusivement sur les conséquences immédiates de ces mouvements (comportements réactifs), on substitue une stratégie dans laquelle les conséquences d'un mouvement sont évaluées au regard des autres mouvements qu'il rend ultérieurement possibles " (comportements prémédités) (Costermans, 2001, p. 106). Cela implique un certain effort de traversée pour rejoindre des lieux aux ambiances attractives. Dans ce cas, il n'est pas systématiquement 
possible d'établir un lien entre l'ambiance locale et la fréquentation d'un espace car celuici peut être emprunté non pas pour sa qualité intrinsèque mais pour la connexion qu'il permet avec des lieux recherchés.

L'environnement urbain est donc mis en mouvement par les individus mobiles. S'il s'impose partiellement aux piétons, ces derniers gardent la possibilité de choisir certains lieux et ambiances auxquels ils seront exposés. Les choix des lieux fréquentés, des itinéraires empruntés constituent une appropriation de l'espace qui peut permettre de révéler les attracteurs d'ambiances individuelles et collectives (Woloszyn et al., 2011), le lien affectif aux espaces tout en les confrontant aux intentions des concepteurs (marqueurs d'ambiances). Partant d'une structure urbaine qui offre un champs des possibles et que l'on doit caractériser, le piéton va tirer une logique d'action qui lui est propre; il va sélectionner et relier les espaces parmi un ensemble de solutions potentielles... Ce continuum, cet art de combiner les lieux entre eux relèvent de la relation homme mobile - espace urbain. En fonction d'où l'on vient et où l'on va, les modulations d'ambiances (visuelles, sonores...) par les cheminements vont se construire tantôt par rupture, tantôt par « accoutumance " progressive, ce que J.-P. Thibaud nomme la « valeur articulatoire du geste» (Thibaud, 2002, p. 186). A ce titre, il pourrait être intéressant de calculer des indices d'entropie ou d'autocorrélation pour chaque itinéraire potentiel afin d'évaluer le comportement des piétons sur ces questions.

\section{Conclusion, la question de l'aménagement de l'espace}

37 En aménagement, on cherche traditionnellement à valoriser l'espace. Mais comment concevoir un environnement urbain perçu de manière positive par la majorité des citadins? Est-ce seulement possible? La manière dont l'espace est agencé, la configuration spatiale peuvent-elles donner naissance à des espaces sensibles attractifs ? $\mathrm{H}$. Lennard propose le concept de ville habitable dans laquelle l'espace public et la pratique de la marche tiennent un rôle prépondérant en offrant l'occasion d'être ensemble, en permettant les rencontres; c'est un lieu d'apprentissage social et de socialisation (Lennard, 1997). Dans cette ville, les considérations esthétiques, la beauté de l'environnement physique (Wong \& Domroes, 2005) sont primordiales. On le pressent bien dans cette description, au-delà de l'environnement construit c'est l'espace habité et pratiqué qui doit focaliser l'attention des aménageurs (Whatmore \& Hinchliffe, 2002).

Or, il subsiste pour le moment une divergence assez forte entre la conception des lieux et le ressenti des habitants ou des usagers. Celle-ci peut être le reflet d'un manque de considération pour les perceptions et représentations habitantes (Moser \& Weiss, 2003 ; Tiano, 2010) ou, plus sûrement, la matérialisation d'une erreur d'appréciation des aménageurs qui manipulent difficilement la question des configurations spatiales des ambiances urbaines. Cette disjonction entre conçu et perçu a engendré une forme de simplification de la production de l'espace, souvent involontaire, mais contre laquelle il faut s'élever. Dans cette optique, il faut soutenir la mise en place de démarches architecturales et urbanistiques dans lesquelles le ressenti des individus est davantage reconnu et pris en compte au regard des espaces sensibles auxquels ils sont soumis. A cet effet, la modélisation des choix individuels de fréquentation des espaces conduit à établir, par exemple, un lien entre des indicateurs mesurables, objectifs et des ambiances privilégiées du point de vue des usages même si une part non négligeable des comportements est informelle, non maîtrisable et non prévisible (Besse, 2010). Les 
résultats que peut fournir un modèle ambiantal de choix constituent un élément très intéressant mais à manipuler avec précaution. En effet, l'approche désagrégée permet de prendre en compte les contraintes de la configuration spatiale des ambiances pour présenter des résultats qui mettent en évidence les choix et donc, selon l'hypothèse retenue, les préférences des individus. Mais il faut par ailleurs rester très prudent car il est impossible de généraliser les résultats obtenus et de les proposer à l'aménagement en dehors du contexte spatial dans lequel ils ont été calculés.

On insistera alors davantage sur la nécessité de contextualiser d'un point de vue spatial les ambiances ce qui permettra d'éviter le piège de la définition d'une forme ou d'une morphologie type. Dans la notion d'ambiance, il n'y a pas de jugement esthétique : même si la qualité d'un lieu peut être reconnue, celle-ci n'est à notre sens valable que dans le cadre très spécifique de sa territorialité, des espaces multi-échelles dans lesquels il s'inscrit. Tout se joue dans la comparaison des espaces les uns par rapport aux autres, et l'on rappellera que l'attractivité d'un lieu s'accompagne de son double négatif pour lequel s'exerce, si ce n'est une répulsion, au moins un déficit en termes de perception et de fréquentation. Dans ce mécanisme de valorisation/ dévalorisation sensorielle des espaces, l'une des questions majeures relève alors de l'harmonisation des ambiances à une échelle mésologique pour éviter des déséquilibres trop prégnants. Cette question de l'harmonisation reste cependant à préciser. Faut-il garantir une certaine diversité des ambiances? Doit-on proposer des portions d'espace présentant des ambiances relativement homogènes? Si oui, à quelle échelle? Faut-il par ailleurs hiérarchiser l'attention que nous portons aux lieux en tenant compte des notions de centralité, d'accessibilité qui confèrent une importance différentielle aux espaces dans la ville?

Autant de questions auxquelles il faut s'efforcer de répondre car si l'aménageur est contraint de travailler sur un espace relativement figé il doit, autant que possible, proposer une structure urbaine qui garantisse une large variété de choix c'est-à-dire dans laquelle des variations, des gradations ou des continuités d'ambiances le long des parcours sont possibles. Cela signifie que la question des ambiances urbaines, notamment dans l'optique d'une redéfinition des modalités de conception des espaces publics urbains, doit passer par un questionnement spatial des ambiances à différentes échelles. En effet, l'organisation spatiale des formes urbaines, des paysages et des ambiances associés est prégnante dans la perception de l'environnement urbain (Lussault, 2000). On pourra même avancer, en guise de perspective, qu'il serait souhaitable de confronter les ambiances perçues ici aux représentations d'une partie des espaces urbains ailleurs (Hégron \& Torgue, 2010). Ces ailleurs constituent des portions d'espace fonctionnant à des échelles et à des métriques différentes (Offner, 2000), la plupart du temps disjointes, et que l'homme considère comme son environnement (Schmitz, 2001). Les limites de cet environnement sont très fluctuantes dans le temps et l'espace pour un individu donné mais elles sont essentielles pour comprendre la perception des ambiances au niveau local et les usages qui en résultent. 


\section{BIBLIOGRAPHIE}

Remerciements :

L'auteur remercie les relecteurs anonymes de la revue pour leurs remarques et suggestions très pertinentes qui ont permis d'améliorer cet article.

Amar, Georges. 1993. Pour une écologie urbaine des transports. Les annales de la recherche urbaine. n' 59-60, p. $140-151$.

Anselin, Luc. 1995. Local Indicators of Spatial Association - LISA. Geographical Analysis. vol. 27, n

- 2, p. 93-115.

Audas, Nathalie \& Martouzet, Denis. 2008. L'affectif urbain. Proposition originale par la cartographie de réactivation des discours. In : Colloque Penser la ville - Approches comparatives. 25-26 octobre, Kenchela, Algérie. p. 62-75

Augoyard, Jean-François. 1979. Pas à pas, essai sur le cheminement quotidien en milieu urbain. Paris : Ed. du Seuil.

Berque, Augustin. 1995. Espace, milieu, paysage, environnement. In : Antoine Bailly; Robert Ferras \& Denise Pumain (eds.). Encyclopédie de géographie. Paris : Economica. p. 349-367.

Besse, Jean-Marc. 2010. Le paysage, espace sensible, espace public. META: Research in Hermeneutics, Phenomenology, and Practical Philosophy. vol. 2, n² 2, p. 259-286.

Brossard, Thierry \& Joly, Daniel. 2004. Analyse quantitative des paysages : concepts, méthodes et applications. In : Emmanuel Raynard \& Jean-Pierre Pralong (eds.). Paysages géomorphologiques. Lausanne : Institut de géographie. p. 21-34.

Brunet, Roger; Ferras, Robert \& Théry, Hervé. 1992. Les mots de la géographie, dictionnaire critique. Paris : La Documentation française.

Caron, Claude \& Roche, Stéphane. 2001. Vers une typologie des représentations spatiales. L'Espace géographique. vol. 30, n 1, p. 1-12.

Cauvin, Colette. 1999. Pour une approche de la cognition spatiale intra-urbaine, Cybergeo: European Journal of Geography [En ligne], Politique, Culture, Représentations, article 72, mis en ligne le 27 janvier 1999, consulté le 03 février 2013. URL : http://cybergeo.revues.org/5043 ; DOI : $10.4000 /$ cybergeo. 5043

Costermans, Jean. 2001. Les activités cognitives. Raisonnement, décision et résolution de problèmes. Bruxelles : Ed. De Boeck Université.

Depraz, Samuel. 2005. Le concept d'Akzeptanz et son utilité en géographie sociale. Exemple de l'acceptation locale des parcs nationaux allemands. L'espace géographique. vol. 34, n 1, p. 1-16.

Di Méo, Guy. 1991. L'Homme, la société, l'espace. Paris : Anthropos.

Foltête, Jean-Christophe \& Piombini, Arnaud. 2010. Deviations in pedestrian itineraries in urban areas: a method to assess the role of environmental factors. Environment and Planning, Part B: Planning and Design. vol. 37, $\mathrm{n}^{\circ}$ 4, p. 723-739.

Goffman, Erving. 1973. La mise en scène de la vie quotidienne. Paris : Les Editions de Minuit. 
Golledge, Reginald G. 1999. Human wayfinding and cognitive maps. In: Reginald G. Golledge (ed.). Wayfinding behaviour, Cognitive mapping and other spatial processes. Baltimore: John Hopkins University Press. p. 5-45.

Golledge, Reginald G. \& Stimson Robert J. 1997. Spatial behavior: a geographic perspective. New-York; London: Guilford Press.

Gourdon, Jean-Loup. 2001. La rue : essai sur l'économie de la forme urbaine. La Tour d'Aigues : Ed. de l'Aube.

Hégron, Gérard \& Torgue, Henry. 2010. Ambiances architecturales et urbaines : de l'environnement urbain à la ville sensible. In : Olivier Coutard \& Jean-Pierre Lévy (eds.). Ecologies urbaines. Paris : Economica. p. 184-197.

Hillier, Bill. 1996. Space is the machine: a configurational theory of architecture. Cambridge: Cambridge University Press.

Hillier, Bill \& Hanson, Julienne. 1984. The social logic of space. Cambridge: Cambridge University Press.

Labussière, Olivier 2009. Les stratégies esthétiques dans la contestation des projets d'aménagement : le milieu géographique entre singularité et exception. L'information géographique . vol. $73, \mathrm{n}^{\circ} 2$, p. 68-88.

Lennard, Suzanne H. Crowhurst; Ungern-Sternberg, Sven von \& Lennard Henry L. (eds). 1997. Making Cities Livable=Wege zur menschlichen stadt. Carmel: Gondolier Press.

Loiseau, Jacques-Marie ; Terrasson, François \& Trochel, Yves. 1993. Le paysage urbain. Paris : Ed. Sang de la terre.

Moles, Abraham \& Rohmer Elisabeth. 1982. Labyrinthes du vécu : l'espace, matière d'actions. Paris : Ed. Librairie des méridiens.

Lussault, Michel. 2000. La ville des géographes. In : Thierry. Paquot ; Michel Lussault \& Sophie Body-Gendrot (eds.). La ville et l'urbain, l'état des savoirs. Paris : Ed. La découverte. p. 21-35.

Lynch, Kevin. 1969. L'image de la cité. Paris : Dunod.

McFadden, Daniel. 1974. Conditional logit analysis of qualitative choice behaviour. In: Paul Zarembka (ed). Frontiers in econometrics. New-York: Academic Press. p. 105-142.

Merleau-Ponty, Maurice. 1942. La structure du comportement. Paris : PUF.

Moser, Gabriel \& Weiss, Karine. 2003. Espaces de vie. Aspects de la relation homme-environnement. Paris : Armand Colin.

Offner, Jean-Marc. 2000. Pour une géographie des interdépendances. In : Jacques Lévy \& Michel Lussault (eds.). Logiques de l'espace, esprit des lieux. Paris : Belin. p. 217-239.

Paulet, Jean-Pierre. 2002. Les représentations mentales en géographie. Paris : Anthropos.

Péneau, Jean-Pierre. 2000. Les ambiances urbaines. In : Marie-France Mattei \& Denise Pumain (eds.). Données urbaines 3. Paris : Anthropos. p. 375-386.

Sansot, Pierre. 1973. Poétique de la ville. Paris : Klincksieck.

Santos, Milton. 1997. La nature de l'espace : technique et temps, raison et émotion. Paris : L'Harmattan.

Schmitz, Serge. 2001. La recherche de l'environnement pertinent. Contribution à une géographie du sensible. L'Espace géographique. vol. 30, n 4, p. 705-735.

Thibaud, Jean-Paul. 2002. L'horizon des ambiances urbaines. Communications. vol. 73, p. 185-201. 
Thomas, Rachel. 2004. Quand le pas fait corps et sens avec l'espace. Aspects sensibles et expressifs de la marche en ville. Cybergeo: European Journal of Geography [En ligne], Dossiers, 3ème colloque du Groupe de Travail Mobilités spatiales et fluidité sociale (GT23) : Offre urbaine et expériences de la mobilité, Strasbourg, France 20-21 et 22 mars 2003. Articles sélectionnés par Cybergeo, article 261, mis en ligne le 01 mars 2004, consulté le 03 février 2013. URL : http:// cybergeo.revues.org/4304; DOI : 10.4000/cybergeo.4304

Tiano, Camille. 2010. Quelles valeurs pour revaloriser les territoires urbains ? Cybergeo: European Journal of Geography [En ligne], Débats, Les valeurs de la ville, mis en ligne le 03 mai 2010, consulté le 04 février 2013. URL : http://cybergeo.revues.org/23091 ; DOI : 10.4000/cybergeo.23091

Whatmore, Sarah \& Hinchliffe, Steve. 2002. Living cities: Making space for urban nature. Soundings: A Journal of Politics and Culture. vol. 22, p. 35-48.

Woloszyn, Philippe; Albisser, Eric; Depeau, Sandrine; Leduc, Thomas; Luckel, Frédéric \& Piombini, Arnaud 2011. PirVE Ambioflux. Caractérisation interdisciplinaire et intersectorielle des attracteurs paysagers des ambiances piétonnes du quartier de Hautepierre à Strasbourg. ESO Travaux et documents, $\mathrm{n}^{\circ} 31$, p. 7-17

Wong, Koon-Kai \& Domroes, Manfred. 2005. The visual quality of urban park scenes of Kowloon Park, Hong Kong: likeability, affective appraisal, and cross-cultural perspectives. Environment and Planning B: Planning and Design. vol. 32, n 4, p. 617-632.

\section{RÉSUMÉS}

Cet article a pour but de présenter le point de vue d'un géographe sur l'organisation spatiale des ambiances urbaines. Au-delà des méthodes de qualification sensible des lieux, le principal thème traité réside dans la caractérisation du contexte spatial des ambiances selon plusieurs méthodes d'analyse spatiale. Les modèles de choix permettent ensuite de confronter cette structure spatiale aux pratiques de mobilité pédestre pour mettre en évidence les préférences ambiantales des citadins. L'objectif est ici de replacer les ambiances dans le jeu complexe d'opportunités et de contraintes qui lie les citadins aux espaces urbains et qui affecte, in fine, leur perception et leurs usages des lieux.

This paper aims to show a geographical point of view about the spatial organization of ambient environment. The main goal consists to describe several methods of spatial analysis in order to characterize the ambient environment spatial context. A choice model can be used to work on both spatial structure and pedestrian mobility experiences and thereby highlight ambient environment preferences of walkers. The objective is to replace ambient environment in the context of opportunities and constraints that bind townspeople to urban areas and ultimately affect people's perceptions and uses of places.

\section{INDEX}

Keywords : spatial structures, context of choice, ambient environment, mobility behaviour, discrete choice models

Mots-clés : organisation spatiale, contexte de choix, ambiances, comportements de mobilité, modèles de choix discrets 


\section{AUTEUR}

\section{ARNAUD PIOMBINI}

Maître de conférences en géographie

Université de Strasbourg, Laboratoire Image, Ville, Environnement

CNRS, ERL 7230 